\title{
Оптимизация составной генераторной ветви
}

\author{
() И.А. Драбкин, В.Б. Освенский \\ АО „Гиредмет“, \\ 119017 Москва, Россия \\ E-mail: igordrabk@gmail.com
}

(Получена 31 января 2017 г. Принята к печати 8 февраля 2017 г.)

Предложен точный способ расчета составной генераторной термоэлектрической ветви в одномерном приближении методом тепловых балансов с эффективными значениями термоэлектрических параметров. Данный метод позволяет точно учесть температурные зависимости электропроводности, коэффициента термоэдс и теплопроводности. Учтено влияние контактных сопротивлений на результирующую эффективность преобразования энергии. Приведен порядок расчета секционированной ветви и показан способ перехода от расчета ветви к расчету термоэлемента.

DOI: $10.21883 /$ FTP.2017.08.44786.55

Для термоэлектрических генераторов, работающих при большой разности температур, секционирование ветвей является естественным способом повышения кпд ветви, поэтому этой теме посвящено довольно много работ [1]. При этом не всегда ясно, из каких соображений получены приводимые кпд и насколько полно проведена оптимизация секционирования. Наиболее последовательным методом оптимизации термоэлектрических устройств является метод максимума Понтрягина [2]. Оптимизация составных генераторных ветвей рассматривается в работе [3], однако процедура оптимизации проведена, на наш взгляд, не совсем корректно. В настоящей работе содержится обобщение метода эффективных тепловых балансов, позволяющее точно решать любую задачу оптимального построения термоэлектрических генераторов.

Для описания процессов внутри ветви надо решить уравнение

$$
(\nabla, \kappa \nabla T)+(\mathbf{j}, \mathbf{j}) \rho-T(\mathbf{j}, \nabla \alpha)=0
$$

где $\kappa$ - теплопроводность, $T-$ температура ветви, j - плотность тока, $\rho$ - удельное сопротивление, $\alpha-$ коэффициент термоэдс. Для определенности, что не ограничивает общности, рассматривается $p$-ветвь, состоящая из $N$ секций. Нумерация секций и координаты границ ясны из рисунка. В одномерном случае, если ось $x$ направлена от холодного конца к горячему, плотность тока $j$ и плотность теплового потока $Q$ отрицательны (см. рисунок).

В уравнениях теплового баланса эти величины положительны, поэтому будем считать $j=-j$ и $Q=-Q$. Уравнение (1) распадается на отдельные уравнения для каждой из секций:

$$
\frac{d}{d x_{i}}\left(\kappa_{i} \frac{d T_{i}}{d x_{i}}\right)+j^{2} \rho_{i}+T_{i} j \frac{d \alpha_{i}}{d x_{i}}=0, \quad i=1,2, \ldots, N
$$

где $T-$ температура, $\rho-$ электропроводность, $\kappa-$ теплопроводность, $\alpha-$ коэффициент термоэдс, $x$ - координата, индекс $i$ - номер секции. Далее для величин с двойным индексом номер секции будем отделять от других индексов запятой.

В качестве граничных условий для ветви длиной $L$ и сечением $s$ возьмем температуры на горячем $\left(T_{h}\right)$ и на холодном $\left(T_{c}\right)$ концах ветви, $\Delta T=T_{h}-T_{c}$. Пусть на горячий конец поступает тепловой поток плотностью $Q_{1}$, а с холодного снимается поток плотностью $Q_{2}$. Граничные условия на концах ветви необходимо дополнить условиями на границе секций. Будем считать, что скачка температур на границе секций нет и $T_{c, i}=T_{h, i-1}, i=2, \ldots, N$, а плотность теплового потока, выходящего из $i$-той секции, поступающего в секцию $i-1$, увеличивается на теплоту Джоуля, выделяющуюся на контактном сопротивлении: $Q_{n, i-1}=Q_{c, i}+j^{2} R_{\text {cont }}, i=2, \ldots, N$. Для решения систему уравнений (2) удобно привести к системе уравнений первого порядка:

$$
\begin{gathered}
\frac{d T_{i}}{d x}=-\frac{\alpha_{i} T_{i} j}{\kappa_{i}}+\frac{q_{i} j}{\kappa_{i}}, \\
\frac{d q_{i}}{d x}=-\frac{\alpha_{i}^{2} T_{i} j}{\kappa_{i}}+\frac{\alpha_{i} q_{i}}{\kappa_{i}}-j \rho_{i}, \quad i=1,2, \ldots, N,
\end{gathered}
$$

где $q_{i}=Q_{i} / j$. Уравнение (3) отличается от приведенного в работе [1] знаком перед $j$.

В каждой секции определим систему эффективных параметров $\alpha_{h . \mathrm{eff}, i}, \rho_{h . \mathrm{eff}, i}, \alpha_{c . \mathrm{eff}, i}, \rho_{c \text {.eff }, i}, \bar{\kappa}_{i}$ [4], с которыми можно записать уравнения теплового баланса

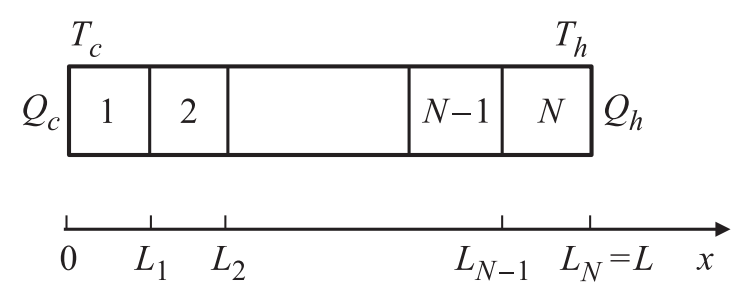

Схема составной термоэлектрической генераторной ветви. 
для каждой секции

$$
\begin{gathered}
Q_{c, i}=\alpha_{c . \mathrm{eff}, i} T_{c, i} j+j^{2}\left(L_{i}-L_{i-1}\right) \rho_{c . \mathrm{eff}, i}+\frac{\bar{\kappa}_{i} \Delta T_{i}}{L_{i}-L_{i-1}}, \\
Q_{h, i}=\alpha_{h . \mathrm{eff}} T_{h, i} j+j^{2}\left(L_{i}-L_{i-1}\right) \rho_{h . \mathrm{eff}, i}-\frac{\bar{\kappa}_{i} \Delta T_{i}}{L_{i}-L_{i-1}} .
\end{gathered}
$$

Выражения для эффективных значений параметров секций с учетом контактных сопротивлений $R_{\text {cont, } i}$ имеют вид [3]:

$$
\begin{aligned}
& \bar{\kappa}_{i}=\frac{L_{i}-L_{i-1}}{\int_{L_{i-1}}^{L_{i}} \frac{d x}{\kappa_{i}\left[T_{i}(x)\right]}} \\
& \alpha_{c . \mathrm{eff}, i}=\alpha_{i}\left(T_{c, i}\right)+\frac{1}{T_{c, i}} \int_{L_{i-1}}^{L_{i}} T_{i} \frac{d \alpha_{i}\left(T_{i}\right)}{d T_{i}} \frac{d T_{i}(y)}{d y} \hat{R}_{i}(y) d y, \\
& \alpha_{h . \mathrm{eff}, i}=\alpha\left(T_{h, i}\right)-\frac{1}{T_{h, i}} \int_{L_{i-1}}^{L_{i}} T_{i} \frac{d \alpha_{i}\left(T_{i}\right)}{d T_{i}} \frac{d T_{i}(y)}{d y} \hat{L}_{i}(y) d y, \\
& \bar{\alpha}_{i}=\frac{1}{T_{h, i}-T_{c, i}} \int_{T_{c, i}}^{T_{h, i}} \alpha_{i}(T) d T \\
& \rho_{h . \mathrm{eff}, i}=\frac{1}{L_{i}-L_{i-1}} \int_{L_{i-1}}^{L_{i}} \rho_{i}\left[T_{i}(y)\right] \hat{L}_{i}(y) d y+\frac{R_{\mathrm{cont}}}{L}, \\
& \rho_{c . \mathrm{eff}, i}=\frac{1}{L_{i}-L_{i-1}} \int_{L_{i-1}}^{L_{i}} \rho_{i}\left[T_{i}(y)\right] \hat{R}(y) d y+\frac{R_{\mathrm{cont}}}{L}, \\
& \bar{\rho}_{i}=\frac{1}{L_{i}-L_{i-1}} \int_{L_{i-1}}^{L_{i}} \rho_{i}[T(x)] d x
\end{aligned}
$$

где

$$
\hat{R}_{i}(y)=\frac{\bar{\kappa}_{i}}{L_{i}-L_{i-1}} \int_{y}^{L_{i}} \frac{d x}{\kappa_{i}(x)}, \hat{L}_{i}(y)=\frac{\bar{\kappa}_{i}}{L_{i}-L_{i-1}} \int_{L_{i-1}}^{y} \frac{d x}{\kappa_{i}(x)}
$$

и

$$
\begin{gathered}
\alpha_{h . \mathrm{eff}, i} T_{h, i}-\alpha_{c . \mathrm{eff}, i} T_{c, i}=\bar{\alpha}_{i}\left(T_{h, i}-T_{c, i}\right), \\
\rho_{h, \mathrm{eff}, i}+\rho_{c, \mathrm{eff}, i}=\bar{\rho}_{i}+\frac{2 R_{\mathrm{cont}}}{L} .
\end{gathered}
$$

После этого рассчитываются эффективные значения параметров для всей ветви, исходя из эффективных значений параметров секций. Введем обозначения для теплового сопротивления $i$-той секции: $R_{t, i}=\frac{L_{i}-L_{i-1}}{s \bar{\kappa}_{i}}$, для суммарного теплового сопротивления ветви: $R_{t a}=\sum_{i=1}^{i=N} R_{t, i}=\bar{\rho}_{t a} \frac{L}{s}$. Обозначим оператором $\hat{R}_{t a, i}=\sum_{k=i+1}^{k=N} \frac{R_{t, k}}{R_{t a}}$ отношение теплового сопротивления справа от $i$-той секции к $R_{t a}$. Выражение для аналогичного оператора слева от $i$-той секции имеет вид: $\hat{L}_{t a, i}=\sum_{k=1}^{k=i-1} \frac{R_{t, k}}{R_{t a}}$. Очевидно, что $\hat{R}_{t a, i}+\hat{L}_{t a, i}+\frac{R_{t, i}}{R_{t a}}=1$, $\hat{L}_{t a, 1}=0, \hat{R}_{t a, N}=0$. В результате получаем значения эффективных параметров для всей ветви:

$$
\begin{aligned}
\alpha_{h N . \mathrm{eff}}= & \alpha_{N}\left(T_{h, N}\right)-\frac{1}{T_{h, N}} \sum_{i=1}^{i=N}\left(\hat{L}_{t a, i} \Delta_{1, i}+\frac{R_{t, i}}{R_{t a}} \Delta_{2, i}\right) \\
& -\frac{1}{T_{h, N}} \sum_{i=2}^{i=N} \hat{L}_{t a, i} \Delta_{3, i}, \\
\alpha_{c N . \mathrm{eff}}= & \alpha_{1}\left(T_{c, 1}\right)+\frac{1}{T_{c, 1}} \sum_{i=1}^{i=N}\left(\hat{R}_{t a, i} \Delta_{1, i}+\frac{R_{t, i}}{T_{t a}} \delta_{2, i}\right) \\
& +\frac{1}{T_{c, q}} \sum_{i=2}^{i=N} \hat{R}_{t a, i-1} \Delta_{3, i},
\end{aligned}
$$

где

$$
\begin{gathered}
\Delta_{1, i}=\alpha_{i}\left(T_{h, i}\right) T_{h, i}-\alpha_{i}\left(T_{c, i}\right) T_{c, i}-\bar{\alpha}_{i} \Delta T_{i}, \\
\Delta_{2, i}=\left[\alpha_{i}\left(T_{h, i}\right)-\alpha_{h . \mathrm{eff}, i}\right] T_{h, i}, \\
\Delta_{3, i}=\left[\alpha_{i}\left(T_{c, i}\right)-\alpha_{i-1}\left(T_{h, i-1}\right)\right] T_{c, i}, \\
\delta_{2, i}=\alpha_{c . \mathrm{eff}, i} T_{c, i}-\alpha_{i}\left(T_{c, i}\right) T_{c, i} .
\end{gathered}
$$

Для эффективных сопротивлений получаем:

$$
\begin{aligned}
\rho_{h N . \mathrm{eff}}= & \sum_{i=2}^{i=N} \hat{R}_{t a, i} \bar{\rho}_{i}+\sum_{i=1}^{i=N} \hat{R}_{t a, i} \rho_{h . \mathrm{eff}, i} \\
& +\frac{1}{L} \sum_{i=2}^{i=N} \hat{R}_{t a, i}\left(R_{\mathrm{cont}, i-1}+R_{\mathrm{cont}, i}\right), \\
\rho_{c N . \mathrm{eff}}= & \sum_{i=1}^{i=N-1} \hat{R}_{t a, i} \bar{\rho}_{i}+\sum_{i=1}^{i=N} \hat{R}_{t a, i} \rho L_{c . \mathrm{eff}, i} \\
& +\frac{1}{L} \sum_{i=2}^{i=N} \hat{R}_{t a, i}\left(R_{\mathrm{cont}, i-1}+R_{\mathrm{cont}, i}\right) .
\end{aligned}
$$

Имеют место соотношения $\alpha_{h N \text {.eff }} T_{h, N}-\alpha_{c N \text {.eff }} T_{c, 1}=$ $=\bar{\alpha}\left(T_{h, N}-T_{c, 1}\right) \quad$ и $\rho_{h N \text {.eff }}+\rho_{c N . \text { eff }}=\bar{\rho}$, где $\bar{\alpha}$ и $\bar{\rho}-$ средние по ветви значения термоэдс и электропроводности. Решив уравнения (4), можно записать уравнения теплового баланса для плотностей теплового потока для составной ветви соответственно для горячей и холодной сторон:

$$
\begin{gathered}
Q_{h N}=\alpha_{h N . \mathrm{eff}} T_{h, N} j-j^{2} L \rho_{h N . \mathrm{eff}}+\frac{\Delta T}{R_{t a}}, \\
Q_{c N}=\alpha_{c N . \mathrm{eff}} T_{c, 1} j+j^{2} L \rho_{c N . \mathrm{eff}}+\frac{\bar{\kappa} \Delta T}{L} .
\end{gathered}
$$


В работе [4] было показано, что кпд ветви $(\eta)$ имеет вид:

$$
\eta=\frac{\Delta T}{T_{h}(1+\beta)} \frac{M /(1+M)}{\left[1+\frac{(1+M)}{Z T_{h}(1+\beta)}-\frac{\Delta T(1+\gamma)}{2 T_{h}(1+M)(1+\beta)}\right]},
$$

где $\beta=\frac{\alpha_{h N . \mathrm{eff}}}{\bar{\alpha}}-1, \gamma=\frac{\rho_{h N . \mathrm{eff}}}{\bar{\rho}}, Z=\frac{\bar{\alpha}^{2} \bar{\rho}_{t a}}{\bar{\rho}}$.

Плотность тока

$$
j=\frac{\bar{\alpha} \Delta T}{\bar{\rho} L(M+1)} .
$$

Максимальное значение $\eta$ достигается при $M=M_{0}$ :

$$
\begin{gathered}
M_{0}=\sqrt{1+Z\left[T_{h, N}(1+\beta)-\Delta T(1+\gamma) / 2\right]}, \\
\eta_{\max }=\frac{\Delta T}{T_{h}(1+\beta)} \frac{M_{0}-1}{M_{0}+1-\frac{\Delta T(1+\gamma)}{T_{h}(1+\beta)}} .
\end{gathered}
$$

Для достижения максимального значения кпд необходимо подобрать такие размеры секций, чтобы $Z$ ветви было максимально. Так как $Z$ зависит только от средних величин, то знак производной по границе секций легко определить аналитически:

$$
\begin{gathered}
\frac{\partial \bar{\alpha}}{d L_{i}}=\frac{1}{\Delta T \kappa_{i-1}\left(T_{h, i-1}\right)} \\
\times \alpha_{i-1}\left(T_{h, i-1}\right) j\left[-\alpha_{i-1}\left(T_{h, i-1}\right) T_{h, i-1}+q_{i-1}\left(L_{i}^{-}\right)\right] \\
-\frac{1}{\Delta T \kappa_{i}\left(T_{c, i}\right)} \alpha_{i}\left(T_{c, i}\right) j\left[-\alpha_{i}\left(T_{c, i}\right) T_{c, i}+q_{i}\left(L_{i}^{+}\right)\right], \\
\frac{\partial \bar{\rho}}{\partial L_{i}}=\frac{1}{L} \rho_{i-1}\left(T_{h, i-1}\right)-\frac{1}{L} \rho_{i}\left(T_{c, i}\right), \\
\frac{\partial \bar{\rho}_{t a}}{\partial L_{i}}=\frac{1}{L} \rho_{t, i-1}\left(T_{h, i-1}\right)-\frac{1}{L} \rho_{t, i}\left(T_{c, i}\right) .
\end{gathered}
$$

Расчет секционированной ветви проводится следующим образом. Вначале решается система (2), при этом начальные условия для $Q_{c, 1}$ подбираются таким образом, чтобы $T_{h, N}=T_{h}$. Для найденных решений в достаточном количестве точек вычисление интегралов, входящих в уравнения (6-12), не представляет труда. После этого вычисляются эффективные параметры по уравнениям (14-17), кпд и плотность тока, а также производные по границам секций от $Z$ по уравнениям (24-26). Затем границы изменяются в соответствии со знаком производной. Процедура повторяется до достижения необходимой точности. После делается аналогичный расчет для $n$-ветви. Согласование по току достигается изменением сечений ветвей или, если это нецелесообразно, берется среднеарифметическое значение плотности тока при небольшой разности этих величин. При более значительном рассогласовании ищется максимум $Z$ для термоэлемента. Описанная процедура существенно проще в работе и удобнее в отладке, чем описанная в работах $[1,2]$.

Работа выполнена при финансовой поддержке Министерства образования и науки РФ (субсидия № RFMEF157914X0039-14.579.21.0039).

\section{Список литературы}

[1] T. Calliat, J.-P. Fleurial, G.J. Snyder, A. Borshchevsky. Proc. XXth International Conf. on Thermoelectrics (Beijing, China, 2001) p. 282.

[2] Л.И. Анатычук, В.А. Семенюк. Оптимальное управление свойствами термоэлектрических материалов и приборов (Черновцы, Прут, 1992).

[3] Л.И. Анатычук, Л.Н. Вихор. Функционально-градиентные термоэлектрические материалы (Черновцы, Букрек, 2012).

[4] И.А. Драбкин, 3.М. Дашевский. В сб.: Термоэлектрики и их применение (СПб., Россия, 2002) с. 281.

Редактор А.Н. Смирнов

\section{Optimization of segmented generating leg}

\section{I.A. Drabkin, V.B. Osvenskii}

JSC „Giredmet", 119017 Moscow, Russia

Abstract A precise method for calculating the segmented thermoelectric generator legs in one-dimensional approximation by method of thermal balance with effective values of thermoelectric parameters is proposed. This method allows precisely to take into account the temperature dependence of the electrical conductivity, the Seebeck coefficient and thermal conductivity. The effect of the contact resistance on the resulting power conversion efficiency is also taken into account. The way of calculation of the segmented leg and that of the transition from a leg to a couple is shown. 